\title{
Stability on time-dependent domains: convective and dilution effects
}

\author{
R. Krechetnikov ${ }^{\mathrm{a}, *}$, E. Knobloch ${ }^{\mathrm{b}}$ \\ ${ }^{a}$ Department of Mathematics, University of Alberta, Edmonton, AB T6G 2G1, Canada \\ ${ }^{b}$ Department of Physics, University of California, Berkeley, CA 94720, USA
}

\begin{abstract}
In this paper we explore near-critical behavior of spatially extended systems on time-dependent spatial domains with convective and dilution effects due to domain flow. As a paradigm, we use the Swift-Hohenberg equation, which is the simplest nonlinear model with a finite non-zero critical wavenumber, to study dynamic pattern formation on time-dependent domains. A universal amplitude equation governing weakly nonlinear evolution of the pattern on time-dependent domains is derived and proves to be a generalization of the standard Ginzburg-Landau equation. Its key solutions identified here demonstrate a substantial variety spatially periodic states with a time-dependent wavenumber, steady spatially non-periodic states, and pulse-train solutions - in contrast to extended systems on time-fixed domains. The effects of domain flow, such as bifurcation delay due to domain growth and destabilization due to oscillatory domain flow, on the Eckhaus instability responsible for phase slips of spatially periodic states are analyzed with the help of both local and global stability analyses. A nonlinear phase equation describing the approach to a phase-slip event is derived. Detailed analysis of a phase slip using multiple time scale methods demonstrates different mechanisms governing the wavelength changing process at different stages.
\end{abstract}

Keywords: stability theory, Eckhaus instability, time-dependent domains, amplitude equations, pattern formation

\section{Introduction}

\subsection{General setting}

In the present work we consider pattern formation in a general evolution system on a time-dependent domain $\mathbf{x} \in \Omega_{t}$ :

$$
\frac{\partial c}{\partial t}+\nabla \cdot(\mathbf{u} c)=\mathcal{L}_{\mathbf{x}} c+N(c), \quad N(0)=N^{\prime}(0)=0,
$$

resulting from the application of a conservation law to a given quantity $c$ such as a concentration (or concentrations if (1) is a vector equation). Here $\mathcal{L}_{\mathbf{X}}$ is a constant coefficient timeindependent differential operator in the spatial variable $\mathbf{x}$ (in the case of a reaction-diffusion system $\mathcal{L}_{\mathbf{x}}=D \nabla^{2}$, for example); $N(c)$ is a general nonlinear differential operator, which may originate from the nonlinear part of the reaction law; $\mathbf{u}(\mathbf{x}, t)$ is the velocity of a spatial domain point at location $\mathbf{x}$ at time $t$. The evolution of the quantity $c$ is considered on a time-deforming domain $\Omega_{t}$, which can be thought of as a 'substrate': examples include reaction-diffusion on growing skin, crown spike structure on a growing circular rim in the drop splash phenomena, waves in a stretched rod, etc. [1]. The time-deformation of the domain $\Omega_{t}$, which may be finite or infinite in spatial extent, introduces an advection term, $\mathbf{u} \cdot \nabla c$, corresponding to elementary volumes moving with the flow $\mathbf{u}(\mathbf{x}, t)$ due to local domain deformation and a dilution term, $c \nabla \cdot \mathbf{u}$, corresponding to local volume change. 6826

${ }^{*}$ Corresponding author. Phone: +1 (780) 492-1926. Fax: +1 (780) 492-

Email address: krechet@ualberta.ca (R. Krechetnikov)
In addition to this Eulerian interpretation, it is instructive to look at the local flow $\mathbf{u}(t, \mathbf{x})$ from a Lagrangian description point of view. Suppose the point $\mathbf{x}$ in the domain $\Omega_{t}$ moves according to the trajectory $\mathbf{x}=\mathbf{X}(t, \mathbf{a}) \in \Omega_{t}$, where $\mathbf{a}$ is an initial position (point label or a Lagrangian coordinate), i.e. $\mathbf{X}(0, \mathbf{a})=\mathbf{a}$. The local flow is then fully determined by $\mathbf{u}(t, \mathbf{x})=\partial \mathbf{X} / \partial t$, where the partial derivative is evaluated at constant $\mathbf{a}$, i.e. a fixed marker for the given trajectory. We mention two key examples of domain deformation - for a more detailed discussion the reader is referred to [1]. First, isotropic growth, which in one spatial dimension corresponds to $X(t, a)=\operatorname{ar}(t)$ with $r(0)=1$ and $a \in\left[-L_{0} / 2, L_{0} / 2\right]$, implies

$$
u(t, x)=a \dot{r}=x \frac{\dot{r}}{r}=x \frac{\dot{L}}{L} \equiv f(t) x
$$

where $L(t)=L_{0} r(t)$ is the domain length at time $t$; the function $f(t) \equiv \dot{L} / L$ will be used throughout the rest of the paper. Thus the velocity $u$ of stretching depends on the location away from the stationary center $x=0$ with maximum speed at the domain boundaries $\left.u\right|_{x= \pm L(t) / 2}= \pm L_{0} \dot{r} / 2= \pm \dot{L} / 2$. Note that the flow becomes a function of space only, $u=u(x)$, when the domain grows exponentially: $r \sim e^{\alpha t}, \alpha>0$. Pattern formation on exponentially growing domains was studied numerically in a number of papers, e.g. [2, 3, 4, 5, 6]. If each point of the domain oscillates periodically with period $T$, then $X(t, a)=X(t+T, a)$ for all $t$ and the flow $u(t, x)$ is called oscillatory - physically this may correspond to jittering endpoints of the domain (in opposite directions). When all points of the domain move with the same speed $u=u(t)$, the domain is simply translated and no stretching takes place - therefore, in what follows we only 
consider flows $u$ depending on the spatial coordinate $x$ (and perhaps time). An important special case is that of uniform growth of a spatially periodic domain studied in [7], corresponding to a one-dimensional domain that stretches at the same rate everywhere - because the domain does not have endpoints, each cross-section remains at rest and $u \equiv 0$ everywhere. This is the case, for example, during the process of crown formation in the drop splash problem [7], when dilution effect is neglected.

\subsection{Motivation and key questions}

The present study is motivated by the need to understand domain flow (convection and dilution) effects on near-critical behavior and in particular on the Eckhaus instability in various physical systems. The Eckhaus instability is the key instability that permits a system exhibiting a periodic structure with a characteristic length scale to adjust to a growing domain by nucleating new wavelengths to maintain the characteristic scale of the pattern. To date, most knowledge in this area is based on experiments or numerical simulations, as illustrated by models of morphogenesis, e.g. [2, 8, 9, 3, 4]. Reaction-diffusion systems have been studied numerically on domains with both isotropic (including exponential) [2, 4, 6] and nonuniform [8] growth, revealing growth via wavelength-doubling. In [2] the flow term in Eq. (1) was neglected, thereby rendering the system analogous to translation-invariant systems with periodic boundary conditions [7]. Given the wealth of observational data, there is a need for a simple theory testing the basic mechanisms governing structure growth. One approach, based on a reduction of PDEs to ODEs, was proposed in [4] for domains with isotropic growth, but it does not seem to have the same clarity as the classical Eckhaus instability analysis [10]. Other known results about stability on time-dependent domains include the assertion that if the flow $\mathbf{u}$ is divergence-free the conditions for a diffusion-induced (Turing) instability remain unchanged from those for the time-independent case [9].

Given that fundamental understanding of stability properties on time-dependent domains is currently in a rudimentary state, in the present work we address basic questions centered around the effect of the flow $\mathbf{u}(t, \mathbf{x})$ on pattern formation in near-critical systems, in particular:

- Is there a universal near-critical amplitude equation similar to the Ginzburg-Landau equation (GLE) on timeindependent spatial domains?

- How may the spatial structure of solutions be affected by time-evolution of the domain?

- What are the mechanisms by which the pattern wavelength (number of cells) changes and what is the nature of the boundary separating the basins of attraction of solutions with different number of cells compared with the standard case of Eckhaus instability $[11,12]$ ?

The discussion below parallels the corresponding theory for patterns on time-independent domains $[11,12]$ although the results demonstrate substantial differences arising from the presence of the domain flow $\mathbf{u}(t, \mathbf{x})$.

\section{Amplitude equation}

\subsection{Problem statement and basic observations}

We seek to understand the effects of domain growth on systems displaying spatially periodic structures. In reactiondiffusion systems of the form (1) this is only possible in coupled equations, i.e. when $c$ is a vector of at least two concentrations [13]. We therefore turn to a generic scalar equation of forth order exhibiting an intrinsic length scale, the Swift-Hohenberg equation (SHE)

$$
c_{t}+(u c)_{x}=\mu c-\left(\partial_{x}^{2}+k_{0}^{2}\right)^{2} c+N(c),
$$

where $x \in[-L(t) / 2, L(t) / 2]$ and $L=L_{0}$ at $t=0$. Figure 1 shows the space-time evolution resulting from an initial condition in the form of a stationary solution of the SHE on a timeindependent domain with $N(c)=-|c|^{2} c$. The domain is assumed to be growing isotropically as described by Eq. (2). The figure reveals that new wavelengths are continuously injected in order to maintain a state with an intrinsic length scale of order $2 \pi / k_{0}$. The advantage of the SHE compared with a system of reaction-diffusion equations (1) is that both the bifurcation parameter $\mu$ and the intrinsic wavenumber $k_{0}$ appear explicitly, thereby making the derivation more compact without affecting the generality of the resulting amplitude equation - the same equation can be arrived at starting from a general near-critical system with $k_{0} \neq 0$. In fact, the SHE (3) can be reduced to a vector reaction-diffusion form by introducing an auxiliary variable $c^{\prime}=\left(\partial_{x}^{2}+k_{0}^{2}\right) c$ as noted in [12], so that (1) becomes an algebraic-differential system in which the variable $c^{\prime}$ evolves on a much faster time scale compared to that of the master mode $c$.

Equation (3) is considered on a finite domain whose length $L(t)$ is assumed to be large enough to contain many wavelengths $2 \pi / k_{0}$ of the primary instability in the time-independent case. The wavenumber $k_{0}$ sets the intrinsic length scale of the problem, even though the primary instability leads to states with a time-dependent wavenumber $k(t)$, with $k(0)=k_{0}$ corresponding to $L(0)=L_{0}$ at $t=0$ - this will become clear once we set the details of the flow $u(t, x)$. As justified by the choice of the modulational scaling below, the departure of $k(t)$ from $k_{0}$ is assumed to be small and to evolve on slow time and spatial scales. Thus, without loss of generality, we 'delegate' this departure from $k_{0}$ to the modulational wavenumber $k(t)-k_{0}$, i.e. the wavenumber set by the solution of the amplitude equation.

Before deriving the amplitude equation, it is helpful to scale the spatial coordinate with respect to the domain size $L(t), x \rightarrow$ $L(t) x$. In the case of isotropic growth (2) we obtain

$$
c_{t}=[\mu-f(t)] c-\left(\frac{1}{L^{2}(t)} \partial_{x}^{2}+k_{0}^{2}\right)^{2} c+N(c),
$$

where $x \in[-1 / 2,1 / 2]$. Thus isotropic domain growth has two main effects on the dynamics: it modifies the bifurcation parameter, which becomes dynamic, $\mu \rightarrow \mu-f(t)$, and it changes the scale on which spatial diffusion acts. In the particular case of an exponentially growing domain, $f(t) \equiv$ const, the effective bifurcation parameter $\mu-f(t)$ remains stationary. Thus, if 


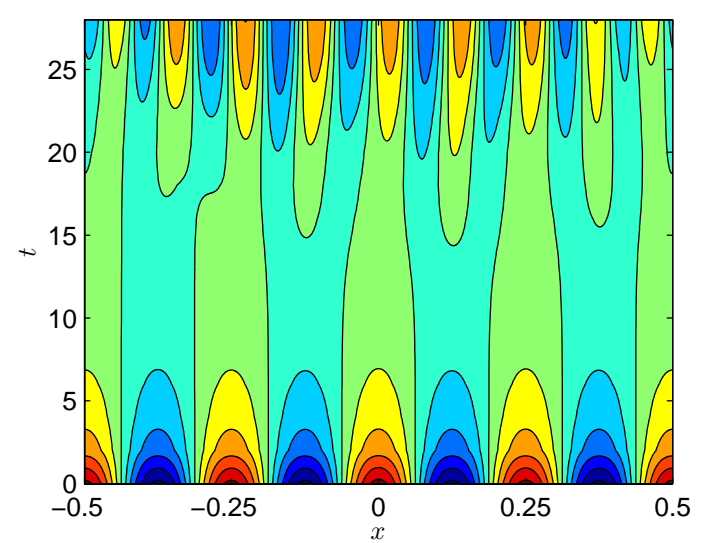

(a)

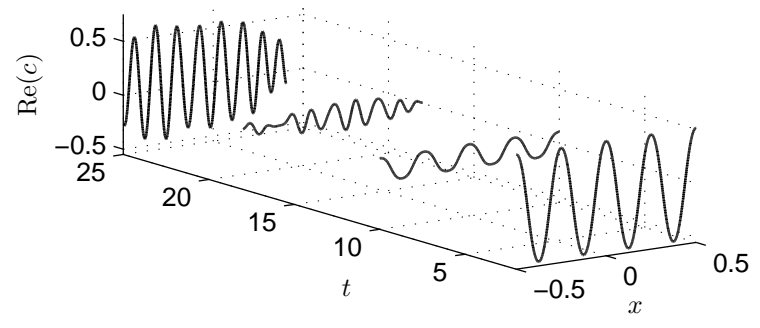

(b)

Figure 1: Phase-slips in the supercritical Swift-Hohenberg equation (3) with $N(c)=-|c|^{2} c$ on an isotropically growing domain with periodic boundary conditions and an initial condition in the form of a stationary pattern with wavenumber $k=4$ and superimposed small amplitude random noise. The parameters are $\mu=0.5, k_{0}=1$ and the domain size expands according to $L(t)=L_{\infty}+\left(L_{0}-L_{\infty}\right) e^{-t / t_{c}}$. (a) A space-time representation of the solution $\operatorname{Re}(c)$. (b) Equidistant contours of $\operatorname{Re}(c)$ in the $(x, t)$ plane. The spatial coordinate is normalized by $L(t)$, so that $x \in[-1 / 2,1 / 2]$ for all $t$. Because of the superimposed noise phase slips occur at different times in different locations.

the domain is being stretched, the effect of stretching is stabilizing since the effective bifurcation parameter is reduced (and potentially may become negative). This observation establishes a direct relation between this type of the domain flow and previous results for translation-invariant systems on time-dependent domains in which only the second effect - the change of the scale on which diffusion operates - is present [7].

\subsection{Derivation}

Let us now consider the SHE (3) in which the flow $u$ is not necessarily isotropic. Since the near-critical amplitude equation describes the dynamics within $O\left(\epsilon^{2}\right)$ of the bifurcation point $\mu=0$ occurring on the slow time scale $\tau=\epsilon^{2} t$ and the long spatial scales $\xi=\epsilon x$, we must assume that the domain growth effects are also weak, specifically $u(t, x)=O\left(\epsilon^{2}\right)$. From a practical viewpoint, consideration of a flow depending on a slow timescale is justified by actual physical considerations, cf. [3].

Given the fast $(t, x)$ and slow $(\tau, \xi)$ temporal and spatial scales, the derivatives in (3) are transformed according to

$$
\partial_{x} \rightarrow \partial_{x}+\epsilon \partial_{\xi}, \quad \partial_{t} \rightarrow \partial_{t}+\epsilon^{2} \partial_{\tau}
$$

In addition we write (same as in the standard GLE derivation)

$$
\mu=\epsilon^{2} \mu_{2}, \quad c=\epsilon c_{1}+\epsilon^{2} c_{2}+\epsilon^{3} c_{3}+\ldots,
$$

together with the assumption $u=\epsilon^{2} u_{2}(\tau, \xi)+\ldots$. Note that $u_{2}$ must, for consistency, be taken to depend on the slow variables only. The above Ansatz transforms (3) into the following system

$$
\begin{aligned}
O(\epsilon): \quad \mathcal{L} c_{1}= & 0 \\
O\left(\epsilon^{2}\right): \quad \mathcal{L} c_{2}= & -4\left(\partial_{x}^{2}+k_{0}^{2}\right) \partial_{x} \partial_{\xi} c_{1}+\frac{1}{2} N^{\prime \prime}(0) c_{1}^{2} \\
O\left(\epsilon^{3}\right): \quad \mathcal{L} c_{3}= & -c_{1 \tau}-\left(u_{2} c_{1}\right)_{x}+\mu_{2} c_{1} \\
& -4\left(\partial_{x}^{2}+k_{0}^{2}\right) \partial_{x} \partial_{\xi} c_{2}-2\left(3 \partial_{x}^{2}+k_{0}^{2}\right) \partial_{\xi}^{2} c_{1} \\
& +N^{\prime \prime}(0) c_{1} c_{2}+\frac{1}{6} N^{\prime \prime \prime}(0) c_{1}^{3}
\end{aligned}
$$

where $\mathcal{L}=\partial_{t}+\left(\partial_{x}^{2}+k_{0}^{2}\right)^{2}$. Equation (7a) is the linear stability problem and admits solutions of the form

$$
c_{1}=R(\tau, \xi) e^{i \phi}+\text { c.c., } \quad \phi(x ; \tau, \xi) \equiv k_{0} x+\int k_{1} \mathrm{~d} \xi .
$$

Without loss of generality $R(\tau, \xi)$ may be taken real since its phase can always be incorporated into $\phi$.

Given the form of (8), Eq. (7b) reduces to

$$
O\left(\epsilon^{2}\right): c_{2 t}+\left(\partial_{x}^{2}+k_{0}^{2}\right)^{2} c_{2}=\frac{1}{2} N^{\prime \prime}(0) c_{1}^{2}
$$

the solution of which can be represented in the form

$$
c_{2}=\alpha_{0} R^{2}+\alpha_{2} R^{2} e^{2 i \phi}+\text { c.c. },
$$

with $\alpha_{0} \equiv \frac{1}{2 k_{0}^{4}} N^{\prime \prime}(0)$ and $\alpha_{2} \equiv \frac{1}{18 k_{0}^{4}} N^{\prime \prime}(0)$. Since

$$
\begin{array}{r}
c_{1 \xi \xi}=\left(R_{\xi \xi}+2 i k_{1} R_{\xi}+i R k_{1 \xi}-R k_{1}^{2}\right) e^{i \phi}+\text { c.c. } \\
\left(\partial_{x}^{2}+k_{0}^{2}\right) \partial_{x} \partial_{\xi} c_{2}=-12 i k_{0}^{3} \alpha_{2}\left(R R_{\xi}+i k_{1} R^{2}\right) e^{2 i \phi}+\text { c.c. }
\end{array}
$$

the (complex) solvability condition for $c_{3}$ obtained from Eq. (7c) leads to the (real) equations

$$
\begin{aligned}
& k_{1 \tau}=8 k_{0}^{2}\left(k_{1} \frac{R_{\xi}}{R}\right)_{\xi}+4 k_{0}^{2} k_{1 \xi \xi}-k_{0} u_{2 \xi}, \\
& R_{\tau}=\left(\mu_{2}-4 k_{0}^{2} k_{1}^{2}\right) R+4 k_{0}^{2} R_{\xi \xi}+\alpha R^{3},
\end{aligned}
$$

where $\alpha \equiv 2 \alpha_{0} N^{\prime \prime}(0)+\frac{1}{2} N^{\prime \prime \prime}(0)$. This system can be combined into a single complex amplitude equation for $A \equiv R e^{i \theta}$ with $\theta_{\xi}=k_{1}$ :

$$
A_{\tau}=\mu A+4 k_{0}^{2} A_{\xi \xi}+\alpha|A|^{2} A-i u k_{0} A
$$

where we dropped the subscripts on $\mu_{2}$ and $u_{2}$. As expected, steady translation of the domain, $u=$ const, leads to the standard GLE via the transformation $A(\tau, \xi) \rightarrow A(\tau, \xi) e^{-i u k_{0} \tau}$, a reflection of the "Doppler" effect. In the following we assume that $\alpha<0$ (so that a supercritical bifurcation takes place) and scale lengths and the amplitude $A$ such that Eq. (13) takes 
the standard form, hereafter referred to as the generalized GLE (gGLE),

$$
A_{\tau}=(\mu-i u) A+A_{\xi \xi}-|A|^{2} A .
$$

Equation (14) will be considered either on an infinite domain or on a domain large enough for Fourier analysis to apply. The gGLE complements the corresponding modulation equations for systems with slowly varying geometry in which the spatial coordinate is time-like, such as occurs, for example, in the sedimentation problem in meandering rivers [14], laser systems with inhomogeneous pumping [15], and flows in spatially developing media [16], to name a few. The key difference is that in such systems the resulting GLE has real coefficients with the bifurcation parameter $\mu$ being a function of the slowly varying spatial variable.

A natural question one may ask is as to why (14) differs from standard GLE if the original SHE (3) on time-dependent domains with a flow can be transformed to the SHE with a dynamic bifurcation parameter (4)? Equation (14) retains translation invariance on the short scale $x$, but this invariance is broken on the larger scale $\xi$. Likewise the term $-i u A$ breaks reflection symmetry, in the sense that Eq. (14) is no longer invariant with respect to the symmetry $A \rightarrow A^{*}$. This is of course a consequence of the odd spatial derivative in Eq. (3) and implies that Eq. (14) no longer has gradient structure. The physical reason for these differences can be traced to the Doppler effect of the flow $u$, which shifts the wavenumber away from $k_{0}$ in (4) and thus affects the phase: the linearized Eq. (3) has the solution $c \propto \exp \left[i k_{0}\left(x-\int_{0}^{\tau} u \mathrm{~d} \tau\right)\right]$. As a result, the flow $u$ is necessarily present in Eq. (12a) for the phase. ${ }^{1}$ It must be noted that one can use the transformation $A \rightarrow A e^{i b(\xi)}$ (e.g. if $u=u(\xi)$ then $\left.b_{\xi \xi}=u\right)$ to remove the imaginary part in the effective bifurcation parameter $\mu-i u$ in (13) but at the expense of changing the bifurcation parameter to $\mu-b_{\xi}^{2}$ and adding a new term $2 i b_{\xi} A_{\xi}$ to the equation.

Equation (14) can also be analyzed in scaled spatial variable $\widetilde{\xi}=\xi L_{0} / L(\tau)$. The resulting equation is defined on a time-fixed spatial domain and takes the form:

$$
A_{\tau}-\frac{L_{\tau}}{L} \widetilde{\xi} A_{\widetilde{\xi}}=(\mu-i u) A+\frac{L_{0}^{2}}{L^{2}} A_{\widetilde{\xi \xi}}-|A|^{2} A .
$$

Equation (15) but with a real coefficient of the first term on the right also arises in control theory $[17,18]$. In the special case where the flow $u$ is time-independent and proportional to $\xi$, Eq. (14) takes, without loss of generality, the form of a nonlinear Airy equation:

$$
\frac{\partial A}{\partial \tau}=(\mu-i \xi) A+\frac{\partial^{2} A}{\partial \xi^{2}}-|A|^{2} A,
$$

which is known to exhibit solutions in the form of pulse trains $[19,20]$.

Since in cases of interest the flow $u(\tau, \xi)$ necessarily depends on the spatial coordinate $\xi$, the nonzero solutions of (14) will in general depend on both space and time.

\footnotetext{
${ }^{1}$ For example, if the domain shrinks exponentially and isotropically, $u=\alpha \xi$, $\alpha<0$, then the deviation of the wavenumber $k=k_{0}+k^{\prime}$ from $k_{0}$ is determined by $k_{0}^{2} k^{\prime 2}+\alpha=0$.
}

\subsection{Spatially periodic unsteady solutions}

Of particular relevance to the main goal of the present paper is a base state whose wavenumber is stretched according to the domain stretching rate. Writing $A_{0}(\tau, \xi)=R_{0}(\tau, \xi) e^{i \theta(\tau, \xi)}$ we find from Eq. (14) that the amplitude $R(\tau, \xi)$ and the wavenumber $k(\tau, \xi) \equiv \theta_{\xi}$ satisfy the equations

$$
\begin{aligned}
k_{\tau} & =2\left(k \frac{R_{0 \xi}}{R_{0}}\right)_{\xi}+k_{\xi \xi}-u_{\xi}=\frac{\partial}{\partial \xi}\left[\frac{1}{R_{0}^{2}}\left(R_{0}^{2} k\right)_{\xi}\right]-u_{\xi}, \\
R_{0 \tau} & =\left(\mu-k^{2}\right) R_{0}+R_{0 \xi \xi}-R_{0}^{3} .
\end{aligned}
$$

A special case of these equations arises when $R_{0}(\tau, \xi)=R_{0}(\tau)$ only. In this case $k(\tau, \xi)$ satisfies the diffusion equation

$$
k_{\tau}=k_{\xi \xi}-u_{\xi}
$$

the solution of which relaxes to a spatially independent wavenumber whenever $u=\xi f(\tau)$. In this case $k_{\tau}=-f(\tau),{ }^{2}$ while the equation for $R_{0}$ gives

$$
R_{0 \tau}=\left[\mu-k^{2}(\tau)\right] R_{0}-R_{0}^{3}
$$

This equation admits the solution

$$
\begin{aligned}
R_{0}^{-2}(\tau) & =F_{0}^{-1}(\tau)\left(2 \int_{0}^{\tau} F_{0}(\tau) \mathrm{d} \tau+R_{0}(0)^{-2}\right), \\
F_{0}(\tau) & =\exp \left(2 \int_{0}^{\tau}\left(\mu-k^{2}(\tau)\right) \mathrm{d} \tau\right)
\end{aligned}
$$

indicating that the amplitude $R_{0}(\tau)$ remains positive at all times provided the initial amplitude $R_{0}(0)>0$ and hence phase slips are necessarily a consequence of phase instability of the base state. Equation (20) also suggests that the system exhibits bifurcation delay [23] since a particular amplitude $R_{0}$ is reached later than in the time-independent domain case with constant bifurcation parameter $\mu-k^{2}$. The resulting base state takes the form

$$
A_{0}(\tau, \xi)=R_{0}(\tau) e^{i \theta(\tau, \xi)}, \quad \theta(\tau, \xi)=k(\tau) \xi+\phi(\tau),
$$

where $\phi(\tau)$ is an arbitrary function of time that will be set to zero in what follows.

\subsection{Spatially non-periodic steady solutions}

Let us now consider time-independent solutions of Eqs. (17) and thus of Eq. (14). Suppose that the spatial dependence occurs on the large scale relative to $\xi$, i.e. $\xi \rightarrow \delta \xi, \delta \ll 1$, and that the flow is correspondingly weak (and time-independent): $u \rightarrow \delta u$. The resulting system simplifies to

$$
\begin{aligned}
& 0=2\left(k \frac{R_{0 \xi}}{R_{0}}\right)_{\xi}+k_{\xi \xi}-u_{\xi}, \\
& 0=\left(\mu-k^{2}\right) R_{0}+\delta^{2} R_{0 \xi \xi}-R_{0}^{3} .
\end{aligned}
$$

\footnotetext{
${ }^{2}$ As the reader can easily establish, the total wavenumber $k_{0}+\epsilon k_{1}$ is commensurate with the changing domain size only approximately, i.e. $\mathrm{d}\left[\left(k_{0}+\epsilon k\right) L\right] / \mathrm{d} \tau=\epsilon k L_{\tau}=O\left(\epsilon^{2}\right)$, which is due to the approximate nature of the near-critical GLE in general. Even on time-fixed spatial domains the solution of the GLE approximates the solution of the original problem with $O\left(\epsilon^{2}\right)$ accuracy only [21, 22].
} 
Expanding $R_{0}$ in powers of $\delta^{2}, R_{0}=R_{00}+O\left(\delta^{2}\right), k=k_{0}+O\left(\delta^{2}\right)$, we obtain

$$
R_{00}^{2}=\mu-k_{0}^{2}
$$

producing the following equation for the leading order wavenumber $k_{0}(\xi)$ :

$$
\frac{\mu-3 k_{0}^{2}}{\mu-k_{0}^{2}} k_{0 \xi}=u
$$

The solutions of the resulting implicit equation

$$
3 k_{0}-\sqrt{\mu} \ln \frac{\sqrt{\mu}+k_{0}}{\sqrt{\mu}-k_{0}}=\int_{0}^{\xi} u(\xi) \mathrm{d} \xi
$$

are shown in Fig. 2 for the case $u(\xi)=\xi$; evidently, Eq. (25) admits multiple solutions, including spatially localized solutions (the two lower branches of $k_{0}(\xi)$ in the figure), and this remains so for other choices of $u(\xi)$ resembling the case $u(\xi)=\xi$ as multivaluedness of $k_{0}$ is defined by the left side of (25). The corresponding amplitude $R_{00}$ found from (23) vanishes when $k_{0}^{2}>\mu$.

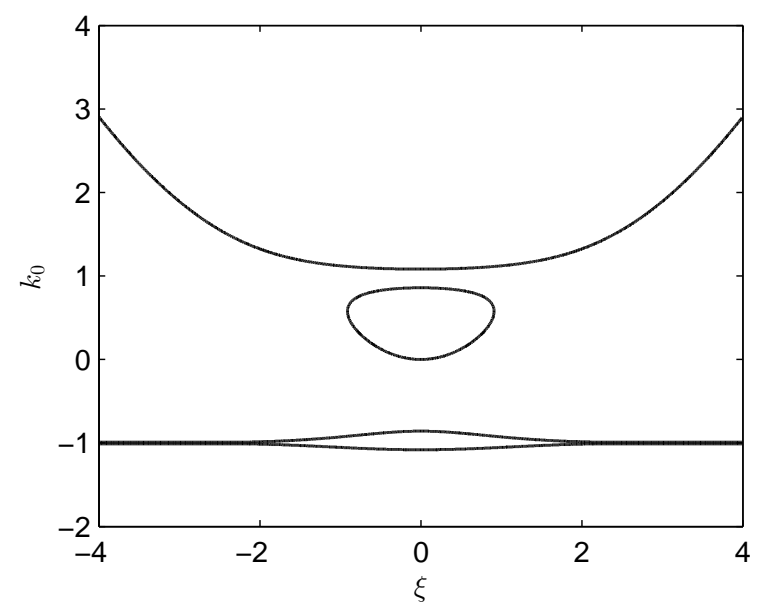

Figure 2: Multivalued solutions $k_{0}(\xi)$ of the implicit equation (25) with $\mu=1$ and $u(\xi)=\xi$.

\section{Eckhaus instability and phase slips}

Among the two key base states - spatially non-periodic steady and spatially periodic unsteady states corresponding to $u=u(\xi)$ and $u=f(\tau) \xi$, respectively - the latter is of particular interest from the point of view of understanding the mechanisms whereby a periodic pattern (with a time-dependent wavenumber) can adjust its wavelength in response to domain growth.

\subsection{Local analysis of a phase-slip event}

A cell or wavelength can only be created or destroyed at locations where the local spatial phase $\theta(\tau, \xi)$ is undefined, i.e., at locations where the order parameter $A$ (Fig. 3(a)) vanishes:

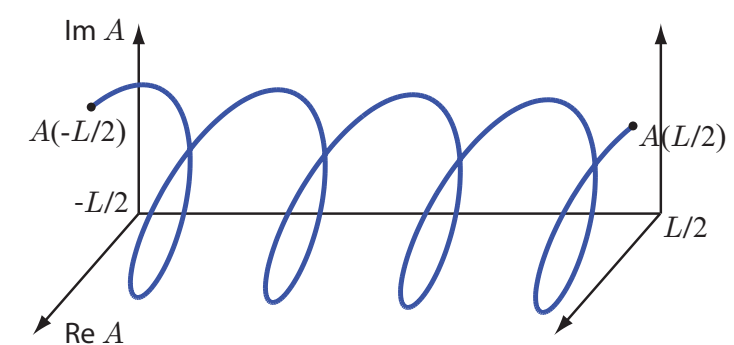

(a)

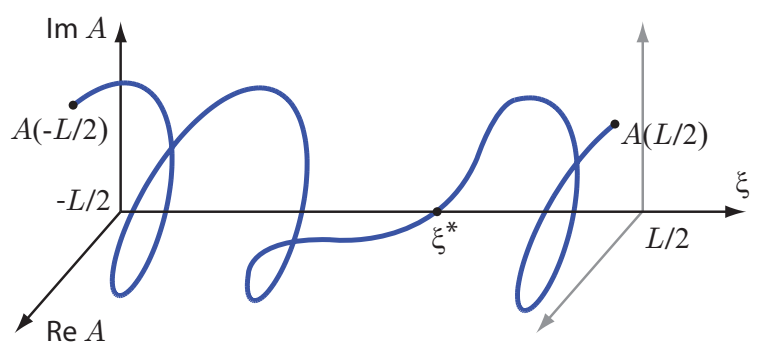

(b)

Figure 3: On a phase-slip event on a time-dependent domain: (a) periodic order parameter $A$; (b) phase slip event at $\left(\widetilde{\tau}^{*}, \widetilde{\xi}_{0}^{*}\right)$.

$\operatorname{Re} A=\operatorname{Im} A=0$ (Fig. 3(b)). Since this is the same scenario as for systems on time-fixed domains, one can apply local analysis [24] to determine the effect of isotropic growth flow $u(\tau, \xi)=f(\tau) \xi$ on the time and location of the phase-slip event.

The key difference between the analysis of [24] and the present case is that here one cannot enforce the phase-slip location to be at $\xi=0$ since the flow breaks translation invariance. However, in the transformed variables introduced in (15) a generic initial condition at $\widetilde{\tau}=0$, right before the phase slip occurs at $(\widetilde{\tau}, \widetilde{\xi})=\left(\widetilde{\tau}^{*}, \widetilde{\xi}_{0}^{*}\right)$, can be written in the form:

$$
A_{0}\left(\widetilde{\xi}-\widetilde{\xi}_{0}^{*}\right)=\frac{1}{2} \alpha\left(\widetilde{\xi}-\widetilde{\xi}_{0}^{*}\right)^{2}+i \beta\left(\widetilde{\xi}-\widetilde{\xi}_{0}^{*}\right)-4 \alpha d_{R}-i \beta d_{I}
$$

where $d_{R}$ and $d_{I}$ are small - the only difference from [24] is the presence of the last term. The structure of (26) reflects the presence of non-zero curvature $\alpha$ and slope $\beta$ at the location of the phase slip, as seen in Fig. 3(b). Representing the solution as

$$
A(\widetilde{\tau}, \widetilde{\xi})=A_{0}\left(\widetilde{\xi}-\widetilde{\xi}_{0}^{*}\right)+\phi(\widetilde{\tau}),
$$

it is easy to show from (15) that

$$
\phi(\widetilde{\tau})=L_{0}^{2} \alpha \int_{0}^{\widetilde{\tau}} \frac{\mathrm{d} \widetilde{\tau}}{L^{2}(\widetilde{\tau})}+i \beta \widetilde{\xi_{0}^{*}} \int_{0}^{\widetilde{\tau}} f(\widetilde{\tau}) \mathrm{d} \widetilde{\tau} .
$$

Hence, the phase slip takes place at $\left(\widetilde{\tau}^{*}, \widetilde{\xi}_{0}^{*}\right)$ defined by

$$
d_{R}=L_{0}^{2} \int_{0}^{\widetilde{\tau}^{*}} \frac{\mathrm{d} \widetilde{\tau}}{L^{2}(\widetilde{\tau})}, \quad d_{I}=\widetilde{\xi}_{0}^{*} \int_{0}^{\widetilde{\tau}^{*}} f(\widetilde{\tau}) \mathrm{d} \widetilde{\tau}
$$

which is in contrast to the conditions for a fixed infinite domain with no flow [24]

$$
\widetilde{\tau}^{*}=d_{R}, \quad \widetilde{\xi_{0}^{*}}=0 .
$$


Equation (29) shows that for $L(\widetilde{\tau})=L(0)+l(\widetilde{\tau})$ with $l(\widetilde{\tau})=s \widetilde{\tau}^{\alpha}$, $\alpha>0$, domain growth $(s>0)$ leads to a delay in the phase slip, while domain contraction $(s<0)$ moves the phase-slip event closer compared to that for the time-independent domain case (30).

As an illustration of an actual phase-slip event in the gGLE on a time-dependent domain, we show in Fig. 4 a sample solution of Eq. (14) on the same isotropically growing domain $L(t)$ as in Fig. 1 exhibiting the instant at which a phase slip takes place, i.e. $|A|=0$.

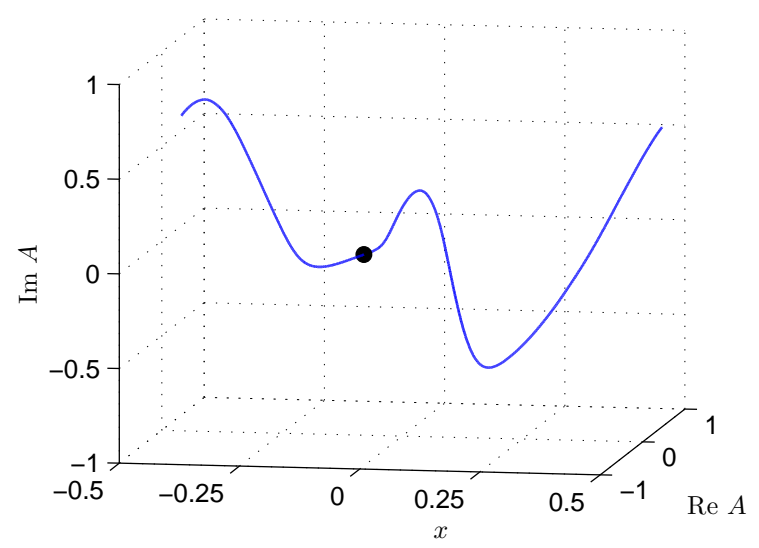

Figure 4: Phase slip in Eq. (14) with $u=f(\tau) \xi$ as derived from the supercritical SHE (3) on the same isotropically growing domain as in Fig. 1. The solid circle marks the instant where $|A|=0$, i.e., the location of the phase slip. The spatial coordinate is normalized by $L(\tau)$ as in (15).

\subsection{Shift in the Eckhaus boundary}

In this section we determine the shift in the Eckhaus boundary arising from the presence of the time-dependent flow $u(\tau, \xi)$. In general this shift cannot be determined analytically. We therefore focus on the particular case $u=f(\tau) \xi$, corresponding to the base state $A_{0}(\tau, \xi)=R_{0}(\tau) e^{i k(\tau) \xi}$ with the time-dependent wavenumber $k(\tau)$, where $k_{\tau}=-f(\tau)$, introduced in $\S 2.3$.

Linear stability properties of this base state are obtained from the linearized equation

$$
A_{\tau}^{\prime}=(\mu-i u) A^{\prime}+A_{\xi \xi}^{\prime}-\left[A_{0}^{2} \bar{A}^{\prime}+2\left|A_{0}\right|^{2} A^{\prime}\right] .
$$

In contrast to the time-independent domain case this equation is in general spatially inhomogeneous. However, when $u=f(\tau) \xi$ (and thus $k_{\tau}=-f(\tau)$ ) solutions of the form

$$
A^{\prime}(\tau, \xi)=a^{+}(\tau) e^{i[k(\tau)-m] \xi}+a^{-*}(\tau) e^{i[k(\tau)+m] \xi}
$$

can be found. Here $m$ is a constant wavenumber. The amplitudes $a^{ \pm}(\tau)$ satisfy

$$
\begin{aligned}
& a_{\tau}^{+}=\left[\mu-(k(\tau)-m)^{2}-2 p(\tau)\right] a^{+}-p(\tau) a^{-}, \\
& a_{\tau}^{-}=\left[\mu-(k(\tau)+m)^{2}-2 p(\tau)\right] a^{-}-p(\tau) a^{+},
\end{aligned}
$$

where $p(\tau) \equiv R_{0}^{2}(\tau)$ is a solution of the Bernoulli equation

$$
\frac{\mathrm{d} p}{\mathrm{~d} \tau}=2\left[\mu-k^{2}(\tau)\right] p-2 p^{2}
$$

The latter equation can be reduced to a linear one via $q(\tau)=$ $1 / p(\tau)$ and thus solved exactly [25]. The result depends on the assumed form of $f(\tau)$ and this in turn determines the stability of the base state $A_{0}(\tau, \xi)$ with respect to sidebands $m \neq 0$. When $k(\tau) \rightarrow k_{0}=$ const as $\tau \rightarrow+\infty$ one recovers the standard Eckhaus boundary $\mu=3 k_{0}^{2}$.

If, however, $k(\tau)$ remains time-dependent in the long-time limit, then the outcome is no longer obvious and the nature of the boundary separating the domain of attraction of solutions with different number of cells is not as simple as in the timeindependent domain case, where the required saddle-point solutions can be constructed explicitly $[11,12]$. We consider here the effect of a time-periodic oscillatory domain flow on the Eckhaus stability boundary in the special case $k(\tau)=k_{0}+\delta \widetilde{\kappa}(\tau)$, where $\widetilde{\kappa}(\tau)=\sin \omega \tau, \delta \ll 1$, and $T=2 \pi / \omega$ is the period. Using standard techniques [26], it can be shown [27] that whenever $k(\tau)$ is $T$-periodic the solution of (34) is also $T$-periodic in the long-time limit. To determine stability of the base state $A_{0}(\tau, \xi)$ we assume that the perturbation wavenumber is also small, $m=\delta \widetilde{m}$, and write

$$
\begin{aligned}
& \mu=\mu_{0}+\delta^{2} \mu_{2}+\delta^{4} \mu_{4}+\ldots \\
& p=p_{0}+\delta p_{1}+\delta^{2} p_{2}+\delta^{3} p_{3}+\delta^{4} p_{4}+\ldots
\end{aligned}
$$

where we took into account that $\mu_{1}=\mu_{3}=0$ since the oscillatory domain flow will produce a nonzero effect at even orders only. The second-order correction $\mu_{2}$ determines the shift in the Eckhaus boundary, i.e., from $\mu_{0}=3 k_{0}^{2}$, arising from the presence of the oscillation $\widetilde{\kappa}(\tau)$. However, as shown below, to compute $\mu_{2}$ it is necessary to go to fourth order in $\delta$. We first solve (34), obtaining the following sequence of linear problems:

$$
\begin{aligned}
\delta^{0}: & \frac{\mathrm{d} p_{0}}{\mathrm{~d} \tau}=2\left[\mu_{0}-k_{0}^{2}\right] p_{0}-2 p_{0}^{2}, \\
\delta^{1}: \quad \frac{\mathrm{d} p_{1}}{\mathrm{~d} \tau} & =2\left[\mu_{0}-k_{0}^{2}\right] p_{1}-4 p_{0} p_{1}-4 k_{0} \widetilde{\kappa} p_{0}, \\
\delta^{2}: \quad \frac{\mathrm{d} p_{2}}{\mathrm{~d} \tau} & =2\left[\mu_{0}-k_{0}^{2}\right] p_{2}-4 p_{0} p_{2}+2\left[\mu_{2}-\widetilde{\kappa}^{2}\right] p_{0} \\
& -4 k_{0} \widetilde{\kappa} p_{1}-2 p_{1}^{2},
\end{aligned}
$$

etc. In each case we seek the solutions as $\tau \rightarrow \infty$. Since $p(\tau)$ is a periodic function of $\tau$ in this limit [27], the $p_{j}$ are all periodic in $\tau$. Specifically, at leading order $p_{0} \rightarrow \mu_{0}-k_{0}^{2}$ as $\tau \rightarrow \infty$, while at $O\left(\delta^{1}\right)$ the long-time solution reads

$$
p_{1}=-4 p_{0} k_{0} \frac{2 p_{0} \sin \omega \tau-\omega \cos \omega \tau}{4 p_{0}^{2}+\omega^{2}} .
$$

Similar expressions can be derived for $p_{j}$ at $O\left(\delta^{j}\right), j=2,3,4$.

The perturbation equations (33) give, at leading order,

$$
\delta^{0}: \mathbf{a}_{0 \tau}=\left(\begin{array}{cc}
\mu_{0}-k_{0}^{2}-2 p_{0} & -p_{0} \\
-p_{0} & \mu_{0}-k_{0}^{2}-2 p_{0}
\end{array}\right) \mathbf{a}_{0},
$$

or simply $\mathbf{a}_{0 \tau} \equiv-p_{0} J_{2} \mathbf{a}_{0}$, where $\mathbf{a}_{i}=\left[a_{i}^{+}, a_{i}^{-}\right]^{T}$ and $J_{2}$ is matrix all of whose elements are 1 . The $O\left(\delta^{1}\right)$ and $O\left(\delta^{2}\right)$ approxima- 
tions are given by

$$
\begin{aligned}
\delta^{1}: \mathbf{a}_{1 \tau} & =-p_{0} J_{2} \mathbf{a}_{1} \\
& -\left(\begin{array}{cc}
\left.2 k_{0} \widetilde{\kappa}-\widetilde{m}\right)+2 p_{1} & p_{1} \\
p_{1} & 2 k_{0}(\widetilde{\kappa}+\widetilde{m})+2 p_{1}
\end{array}\right) \mathbf{a}_{0},
\end{aligned}
$$

and

$$
\begin{aligned}
\delta^{2}: \mathbf{a}_{2 \tau} & =-p_{0} J_{2} \mathbf{a}_{2} \\
& -\left(\begin{array}{cc}
2 k_{0}(\widetilde{\kappa}-\widetilde{m})+2 p_{1} & p_{1} \\
p_{1} & 2 k_{0}(\widetilde{\kappa}+\widetilde{m})+2 p_{1}
\end{array}\right) \mathbf{a}_{1} \\
+ & \left(\begin{array}{cc}
\mu_{2}-(\widetilde{\kappa}-\widetilde{m})^{2}-2 p_{2} & -p_{2} \\
-p_{2} & \mu_{2}-(\widetilde{\kappa}+\widetilde{m})^{2}-2 p_{2}
\end{array}\right) \mathbf{a}_{0},
\end{aligned}
$$

and all can be diagonalized in the same way as the $O\left(\delta^{0}\right)$ system. At $O\left(\delta^{1}\right)$ we obtain

$$
\alpha_{1 \tau}=-p_{0}\left(\begin{array}{cc}
0 & 0 \\
0 & 2
\end{array}\right) \alpha_{1}+\left(\begin{array}{c}
-2 k_{0} \widetilde{\kappa}-p_{1} \\
2 k_{0} \widetilde{m}
\end{array}\right) a_{0}
$$

where $\alpha_{j} \equiv\left[\alpha_{j}, \beta_{j}\right]^{T}$ and $\left.a_{0} \equiv a_{0}^{+}\right|_{\tau=\infty}$ is a constant. This system does not exhibit any secular growth and the resulting longtime asymptotic solution is bounded:

$$
\alpha_{1}=\frac{p_{1}}{2 p_{0}} a_{0}, \quad \beta_{1}=\frac{k_{0} \widetilde{m}}{p_{0}} a_{0} .
$$

At $O\left(\delta^{2}\right)$ we obtain

$$
\left.\alpha_{2 \tau}=a_{0}\left[\mu_{2}-\widetilde{\kappa}^{2}+\widetilde{m}^{2}\right)-p_{2}\right]+2 k_{0} \widetilde{m} \beta_{1}-\left(2 k_{0} \widetilde{\kappa}+p_{1}\right) \alpha_{1} .
$$

We observe that when modulation is absent, i.e., $\widetilde{\kappa}=p_{1}=p_{2}=$ $\mu_{2}=0$, this expression reduces to

$$
\alpha_{2 t}=\left(\frac{3 k_{0}^{2}-\mu_{0}}{\mu_{0}-k_{0}^{2}}\right) \widetilde{m}^{2} a_{0},
$$

and therefore the Eckhaus boundary corresponds to $\mu_{0}=3 k_{0}^{2}$, as expected.

To determine the effect of $\widetilde{\kappa} \neq 0$ on the Eckhaus boundary (i.e., the possible shift $\mu_{2}$ ) we note that at this boundary long wave spatial modulation $(\widetilde{m}>0)$ neither grows nor decays. The requirement that $\alpha_{2}$ is on the marginal stability curve (and thus is a periodic function of $\tau$ with zero mean) imposes the condition

$$
\begin{aligned}
\mu_{2} & =\left\langle\widetilde{\kappa}^{2}+p_{2}\right\rangle-\frac{4 k_{0}^{2} \omega}{\left(4 p_{0}^{2}+\omega^{2}\right)^{2}} \\
& \times\left\langle\left(\omega \cos \omega \tau-2 p_{0} \sin \omega \tau\right)\left(\omega \sin \omega \tau+2 p_{0} \cos \omega \tau\right)\right\rangle,
\end{aligned}
$$

obtained by averaging Eq. (43) over $\tau$. However, Eq. (36c) shows that $p_{0}\left\langle p_{2}\right\rangle=\left(\mu_{2}-\frac{1}{2}\right) p_{0}-2 k_{0}\left\langle\kappa p_{1}\right\rangle-\left\langle p_{1}^{2}\right\rangle$. i.e., $\left\langle p_{2}\right\rangle=\mu_{2}-\frac{1}{2}$. Thus Eq. (45) is an identity and $\mu_{2}$ is undetermined. We therefore proceed to $O\left(\delta^{4}\right)$. The requirement that $\alpha_{4}$ is a periodic function imposes a condition that determines the unknown quantity $\mu_{2}$ (note that $\mu_{4}$ drops out from this condition just like $\mu_{2}$ dropped out at $O\left(\delta^{2}\right)$ ). As a result, we obtain the shift in the Eckhaus boundary:

$$
\mu_{2}=\frac{1}{2}\left(-\widetilde{m}^{2}+\frac{\omega^{4}+64 k_{0}^{4}\left(\omega^{2}+36 k_{0}^{4}\right)}{\left(\omega^{2}+16 k_{0}^{4}\right)^{2}}\right),
$$

which in the long wave limit of interest, $\widetilde{m} \rightarrow 0$, leads to

$$
\mu_{E}\left(k_{0}, \omega\right)=3 k_{0}^{2}+\frac{\delta^{2}}{2} \frac{\omega^{4}+64 k_{0}^{4}\left(\omega^{2}+36 k_{0}^{4}\right)}{\left(\omega^{2}+16 k_{0}^{4}\right)^{2}}+O\left(\delta^{4}\right) .
$$

Since the $O\left(\delta^{2}\right)$ term is positive definite the above result implies that the presence of the oscillatory domain flow is always destabilizing in the sense that the band of stable wavenumbers is always reduced, an effect that increases with decreasing oscillation frequency $\omega$.

In this context, it is interesting to relate these results with those for the case where the control parameter $\mu$ in the Ginzburg-Landau equation is modulated periodically in time [27], viz. $\mu=\bar{\mu}+\delta \mu_{1}, \delta \ll 1$, where $\mu_{1}(\tau)=\sin \omega \tau$. Under these conditions it was found that the band of stable wavenumbers is also always reduced by the presence of the control parameter modulation, with lower frequencies $\omega$ also generating greater reduction: $\mu_{E}\left(k_{0}, \omega\right)=3 k_{0}^{2}+64 k_{0}^{6} \delta^{2}\left(\omega^{2}+16 k_{0}^{4}\right)^{-2}$.

\subsection{Nonlinear analysis: phase equation}

To get an insight into the nonlinear stage close to a phase slip, we start with the gGLE in the standard form (14) on an infinite domain, so that the effect of the domain time-dependence enters through the flow $u(\tau, \xi)$ only. The derivation will be done in the context of stability of spatially periodic unsteady solutions (21) with the amplitude $R_{0}(\tau)$ and wavenumber $k(\tau)$ given by (19) and (18), respectively.

Writing the solution in the form

$$
A(\tau, \xi)=R_{0}(\tau)[1+r(\tau, \xi)] e^{i[k(\tau) \xi+\phi(\tau, \xi)]},
$$

substituting into (14) and separating real and imaginary parts we obtain

$$
\begin{aligned}
r_{\tau} & =r_{\xi \xi}-(1+r)\left(2 k \phi_{\xi}+\phi_{\xi}^{2}\right)-R_{0}^{2}\left(2 r+3 r^{2}+r^{3}\right), \\
\phi_{\tau} & =\frac{2 r_{\xi}\left(k+\phi_{\xi}\right)}{1+r}+\phi_{\xi \xi} .
\end{aligned}
$$

Since we are interested in resolving short time and spatial scales close to a phase slip, we assume that $r(\tau, \xi) \equiv r\left(\tau_{2}, \tau_{4}, \ldots ; \xi_{1}\right)$ and $\phi(\tau, \xi) \equiv \phi\left(\tau_{2}, \tau_{4}, \ldots ; \xi_{1}\right)$, where $\tau=\epsilon^{2} \tau_{2}+\epsilon^{3} \tau_{3}+\epsilon^{4} \tau_{4}+\ldots$ and $\xi=\epsilon \xi_{1}$. With $r=\epsilon r_{1}+\epsilon^{2} r_{2}+\epsilon^{3} r_{3}+\epsilon^{4} r_{4}+\ldots$, Eq. (49a) gives

$$
\begin{aligned}
O(1): & 0 & =-2 k \phi_{\xi_{1}}-2 R_{0}^{2} r_{1}, \\
O(\epsilon): & 0 & =-2 k r_{1} \phi_{\xi_{1}}-\phi_{\xi_{1}}^{2}-R_{0}^{2}\left(2 r_{2}+3 r_{1}^{2}\right), \\
O\left(\epsilon^{2}\right): & r_{1 \tau_{2}} & =r_{1 \xi_{1} \xi_{1}}-2 k r_{2} \phi_{\xi_{1}}-r_{1} \phi_{\xi_{1}}^{2} \\
& & -R_{0}^{2}\left(2 r_{3}+6 r_{1} r_{2}+r_{1}^{3}\right),
\end{aligned}
$$

while the phase equation (49b) produces

$$
\begin{aligned}
O(1): & \phi_{\tau_{2}} & =\phi_{\xi_{1} \xi_{1}}+2 k r_{1 \xi_{1}} \\
O(\epsilon): & \phi_{\tau_{3}} & =2\left(k r_{2 \xi_{1}}-k r_{1} r_{1 \xi_{1}}+\phi_{\xi_{1}} r_{1 \xi_{1}}\right) \\
O\left(\epsilon^{2}\right): & \phi_{\tau_{4}} & =2\left[k r_{3 \xi_{1}}+\phi_{\xi_{1}} r_{2 \xi_{1}}+k r_{1 \xi_{1}}\left(r_{1}^{2}-r_{2}\right)\right. \\
& & \left.-r_{1}\left(k r_{2 \xi_{1}}+\phi_{\xi_{1}} r_{1 \xi_{1}}\right)\right] .
\end{aligned}
$$


These equations describe the initial stages of a phase-slip event. The system (50) leads to the following solutions

$$
\begin{aligned}
& r_{1}=-\frac{k \phi_{\xi_{1}}}{R_{0}^{2}} \\
& r_{2}=-\frac{\phi_{\xi_{1}}^{2}}{2 R_{0}^{2}}\left(1+\frac{k^{2}}{R_{0}^{2}}\right) \\
& r_{3}=\frac{1}{2 R_{0}^{2}}\left[\left(\frac{k}{R_{0}^{2}}\right)_{\tau_{2}} \phi_{\xi_{1}}-2 \frac{k^{3}}{R_{0}^{4}} \phi_{\xi_{1} \xi_{1} \xi_{1}}\right]-\frac{1}{2} \frac{k}{R_{0}^{4}} \phi_{\xi_{1}}^{3}\left(1+\frac{k^{2}}{R_{0}^{2}}\right)
\end{aligned}
$$

while (51) yields

$$
\begin{aligned}
\phi_{\tau_{2}} & =\phi_{\xi_{1} \xi_{1}}\left(1-\frac{2 k^{2}}{R_{0}^{2}}\right), \\
\phi_{\tau_{3}} & =-4 \phi_{\xi_{1}} \phi_{\xi_{1} \xi_{1}}\left(1+\frac{k^{2}}{R_{0}^{2}}\right) \frac{k}{R_{0}^{2}}, \\
\phi_{\tau_{4}}= & \frac{k}{R_{0}^{2}}\left(\frac{k}{R_{0}^{2}}\right)_{\tau_{2}} \phi_{\xi_{1} \xi_{1}}-2 \frac{k^{4}}{R_{0}^{6}} \phi_{\xi_{1} \xi_{1} \xi_{1} \xi_{1}} \\
& -\frac{1}{R_{0}^{2}}\left(1+\frac{k^{2}}{R_{0}^{2}}\right)\left(2+7 \frac{k^{2}}{R_{0}^{2}}\right) \phi_{\xi_{1}}^{2} \phi_{\xi_{1} \xi_{1}} .
\end{aligned}
$$

Equations (53) show that the phase $\phi$ evolves in general on the three distinct timescales $\tau_{2-4}$. Which of these timescales is relevant depends on the distance from the Eckhaus boundary. When $R_{0}^{2}-2 k^{2}=O(1)$ and negative, the dynamics is governed by the diffusion equation (53a) with a negative diffusion coefficient and instability develops on the timescale $\tau_{2}$. When $R_{0}^{2}-2 k^{2}=\epsilon \mathcal{F}\left(\tau_{3}\right)$ the instability grows on the same timescale $\tau_{3}$ as wave steepening, as described by

$$
\phi_{\tau_{3}}=\phi_{\xi_{1} \xi_{1}}\left(1-\frac{2 k^{2}}{R_{0}^{2}}\right)-4 \phi_{\xi_{1}} \phi_{\xi_{1} \xi_{1}}\left(1+\frac{k^{2}}{R_{0}^{2}}\right) \frac{k}{R_{0}^{2}},
$$

but wave steepening alone does not arrest the instability. The growth is slow enough to be arrested only when $R_{0}^{2}-2 k^{2}=$ $\epsilon^{2} \mathcal{F}\left(\tau_{4}\right)$, i.e., sufficiently close to the Eckhaus boundary. Only in this case is the phase $\phi$ defined for all time. To show this we suppose, in addition, that $R_{0}$ and $k$ both evolve on the timescale $\tau_{4}$ and scale the phase $\phi \rightarrow \epsilon \phi$, obtaining

$$
\begin{aligned}
\phi_{\widehat{\tau}}=\left[1-\frac{2 k^{2}}{R_{0}^{2}}+\frac{k}{R_{0}^{2}}\right. & \left.\left.\frac{k}{R_{0}^{2}}\right)_{\widehat{\tau}}\right] \phi_{\overparen{\xi \xi}} \\
& -\frac{4 k}{R_{0}^{2}}\left(1+\frac{k^{2}}{R_{0}^{2}}\right) \phi_{\widetilde{\xi}} \phi_{\widetilde{\xi \xi}}-\frac{2 k^{4}}{R_{0}^{6}} \phi_{\widetilde{\xi \xi \zeta \xi \xi}} .
\end{aligned}
$$

Here $\widehat{\tau}=\tau_{4}$ is the only timescale present and $\widehat{\xi}=\xi_{1}$. The resulting equation - the Kuramoto-Sivashinsky equation with time-dependent coefficients - shows that sufficiently close to the Eckhaus boundary the instability grows too slowly to proceed to completion and is in fact arrested by hyperdiffusion. Indeed, if we neglect the time-dependence and linearize Eq. (55), the growth rate of an infinitesimal disturbance is given by

$$
\sigma=-\left(1-\frac{2 k^{2}}{p}\right) \widetilde{m}^{2}-\frac{2 k^{4}}{p^{3}} \widetilde{m}^{4}=\frac{\widetilde{m}^{2}}{2 k^{2}}\left(3 k^{2}-\mu-\frac{1}{2} \widetilde{m}^{2}\right),
$$

where $\widetilde{m} \ll 1$ is the wavenumber of the perturbation and $p=$ $R_{0}^{2}$. Thus the Eckhaus boundary occurs at $\mu=3 k^{2}-\frac{1}{2} \widetilde{m}^{2}$, a result that agrees precisely with Eq. (46).

In the time-dependent case, the linearization is a nonautonomous ordinary differential equation,

$$
\widehat{\phi}_{\widehat{\tau}}=g(\widehat{\tau}) \widehat{\phi}, \quad g(\widehat{\tau})=-\left[1-\frac{2 k^{2}}{p}+\frac{k}{p}\left(\frac{k}{p}\right)_{\widehat{\tau}}\right] \widetilde{m}^{2}-\frac{2 k^{4}}{p^{3}} \widetilde{m}^{4}
$$

where $\phi(\widehat{\xi}, \widehat{\tau})=\widehat{\phi}(\widehat{\tau}) e^{i \widetilde{m} \widehat{\xi}}$. The solution of this equation is $\widehat{\phi}(\widehat{\tau})=\widehat{\phi}(0) \exp \left(\int_{0}^{\widehat{\tau}} g\left(\widehat{\tau}^{\prime}\right) d \widehat{\tau}^{\prime}\right)$, implying that it grows in time provided $\int_{0}^{\widehat{\tau}} g\left(\widehat{\tau}^{\prime}\right) d \widehat{\tau}^{\prime}>0$. When $k=k_{0}+\delta \sin \omega \widehat{\tau}, \delta \ll 1$, this condition, with the integral taken over one period of the forcing, yields

$$
\mu=3 k_{0}^{2}+\frac{9}{2} \delta^{2}-\frac{1}{2} \widetilde{m}^{2}+O\left(\delta^{4}, \delta^{2} \widetilde{m}^{2}\right)
$$

as the threshold for the Eckhaus instability. This results agrees with the $\omega \rightarrow 0$ limit of Eq. (47), thereby confirming the validity of the explicit stability calculation performed in Sec. 3.2 and the prediction from the phase equation obtained in this section.

We remark that Eq. (57) can also be used to study the effect on the Eckhaus instability of nonperiodic forcing. For example, when the wavenumber $k$ increases linearly with time due to domain compression, $k=k^{\dagger}(1+c \widehat{\tau})$, with $c>0$ and $k(0)=k^{\dagger}$ inside the Eckhaus-stable region (i.e., $g(0)<0$ ), instability sets in at $\widehat{\tau}=\widehat{\tau}_{\text {exit }}$ defined by

$$
\int_{0}^{\widehat{\tau}_{\mathrm{exit}}} g\left(\widehat{\tau}^{\prime}\right) d \widehat{\tau}^{\prime}=0
$$

Since $\widehat{\tau}_{\text {exit }}>\widehat{\tau}^{\dagger}$, where $g\left(\widehat{\tau}^{\dagger}\right)=0$ represents the time at which the function $g(\tau)$ passes from negative to positive (the instantaneous passage through the Eckhaus boundary), we speak of bifurcation delay arising from the presence of time-dependence [7].

\section{Conclusions}

In this work we made some progress in understanding the effects of convection and dilution arising from domain deformation on pattern formation in near-critical systems. First, for isotropic growth the effects of convection and dilution can be transformed away and replaced by a dynamic bifurcation parameter, at least in one spatial dimension. Second, we showed that in general the near-critical evolution of the system is described by an amplitude equation of Ginzburg-Landau type (referred to here as gGLE) but with an additional pure imaginary linear term whose form depends on the details of the domain deformation. These results, in particular, allow us to invoke earlier analyses of systems with a dynamic bifurcation parameter [7] or those with a complex, spatially inhomogeneous linear term $[19,20]$. Third, new solutions, compared to the standard GLE, are found, including spatially periodic solutions with a time-dependent wavenumber and even spatially non-periodic (spatially localized) steady states. Finally, we also examined the properties of the Eckhaus instability as described by the 
gGLE, with a view of elucidating the effects of domain deformation on the occurrence of phase slips whereby the system tries to maintain a preferred wavenumber in the face of domain growth. Local analysis reveals that time-dependence delays the occurrence of a phase slip (compared to the time-independent domain case) when domain is growing. We also performed a linear stability study of spatially periodic wavetrains and derived a nonlinear phase equation of Kuramoto-Sivashinsky type with time-dependent coefficients that captures the initial stages of the phase-slip development. Both analyses show that the effect of an oscillatory domain flow is always destabilizing, i.e., that the band of stable wavenumbers shrinks.

\section{Acknowledgements}

This work was partially supported by the National Science Foundation (NSF) under Collaborative Research Grants CMMI-1232902 and CMMI-1233692, CAREER award under Grant No. 1054267, and the Natural Sciences and Engineering Research Council of Canada (NSERC) under Grant No. 6186.

\section{References}

[1] E. Knobloch, R. Krechetnikov, Problems on time-varying domains: formulation, dynamics, and challenges, Acta Appl. Math. 137 (2015) 123157.

[2] E. J. Crampin, E. A. Gaffney, P. K. Maini, Reaction and diffusion on growing domains: scenarios for robust pattern formation, Bull. Math. Biology 61 (1999) 1093-1120.

[3] A. Madzvamuse, E. A. Gaffney, P. K. Maini, Stability analysis of nonautonomous reaction-diffusion systems: the effects of growing domains, J. Math. Biol. 61 (2010) 133-164.

[4] H. Ueda, A remark on parametric resonance for wave equations with a time periodic coefficient, Proc. Japan Acad. A 87 (2011) 128-129.

[5] J. A. Mackenzie, A. Madzvamuse, Analysis of stability and convergence of finite-difference methods for a reaction-diffusion problem on a onedimensional growing domain, IMA J. Numer. Anal. 31 (2011) 212-232.

[6] K. Ueda, Y. Nishiura, A mathematical mechanism for instabilities in stripe formation on growing domains, Physica D 241 (2012) 37-59.

[7] E. Knobloch, R. Krechetnikov, Stability on time-dependent domains, J. Nonlinear Sci. 24 (2014) 493-523.

[8] E. J. Crampin, W. W. Hackborn, P. K. Maini, Pattern formation in reaction-diffusion models with nonuniform domain growth, Bull. Math. Biology 64 (2002) 747-769.

[9] A. Madzvamuse, Turing instability conditions for growing domains with divergence free mesh velocity, Nonlinear Analysis 71 (2009) e2250e2257.

[10] W. Eckhaus, Studies in non-linear stability theory, Springer-Verlag, New York, 1965

[11] J. S. Langer, V. Ambegaokar, Intrinsic resistive transition in narrow superconducting channels, Phys. Rev. 164 (1967) 498-510.

[12] L. Kramer, W. Zimmermann, On the Eckhaus instability for spatially periodic patterns, Physica D 16 (1985) 221-232.

[13] L. Kramer, E. Ben-Jacob, H. Brand, M. C. Cross, Wavelength selection in systems far from equilibrium, Phys. Rev. Lett. 49 (1982) 1891-1894.

[14] W. Eckhaus, R. Kuske, Pattern formation in systems with slowly varying geometry, SIAM J. Appl. Math. 57 (1997) 112-152.

[15] M. N. Ouarzazi, P. A. Bois, M. Taki, Global-stability analysis of transverse modes in laser systems under inhomogeneous pumping, Phys. Rev. A 53 (1996) 4408-4419.

[16] R. E. Hunt, D. G. Crighton, Instability of flows in spatially developing media, Proc. Roy. Soc. Lond. A 435 (1991) 109-128.

[17] K. Roussopoulos, P. A. Monkewitz, Nonlinear modelling of vortex shedding control in cylinder wakes, Physica D 97 (1996) 264-273.
[18] J. Xiao, G. Hu, J. Yang, J. Gao, Controlling turbulence in the complex Ginzburg-Landau equation, Phys. Rev. Lett. 81 (1998) 5552-5555.

[19] A. P. Bassom, A. M. Soward, On finite-amplitude subcritical instability in narrow-gap spherical Couette flow, J. Fluid Mech. 499 (2004) 277-314.

[20] E. W. Blockley, A. P. Bassom, A. D. Gilbert, A. M. Soward, Pulse-train solutions of a spatially heterogeneous amplitude equation arising in the subcritical instability of narrow gap spherical Couette flow, Physica D 228 (2007) 1-30.

[21] A. van Harten, On the validity of the Ginzburg-Landau equation, J. Nonlinear Sci. 1 (1991) 397-422.

[22] I. Melbourne, Derivation of the time-dependent Ginzburg-Landau equation on the line, J. Nonlin. Sci. 8 (1998) 1-15.

[23] C. Lobry, Dynamic bifurcations, in: Dynamic bifurcations, Springer, 1991, pp. 1-13.

[24] J.-P. Eckmann, T. Gallay, C. E. Wayne, Phase slips and the Eckhaus instability, Nonlinearity 8 (1995) 943-961.

[25] E. Kamke, Handbook of ordinary differential equations, Fizmatgiz, Moscow, 1961.

[26] R. Grimshaw, Nonlinear ordinary differential equations, Blackwell Scientific Publications, Boston, 1990.

[27] J. Moehlis, E. Knobloch, Eckhaus-Benjamin-Feir instability in systems with temporal modulation, Phys. Rev. E 54 (1996) 5161-5168. 